\title{
Ring currents in benzo- and benzocyclobutadieno-annelated biphenylene derivates
}

\author{
Slavko Radenković ${ }^{* a]}$, Jelena Tošović ${ }^{[a]}$, Remco W.A. Havenith ${ }^{[b, c]}$ and Patrick Bultinck ${ }^{[c]}$
}

\begin{abstract}
The effect of benzo- and benzocyclobutadieno-annelation on the current density induced in a series of biphenylene derivates is examined at the B3LYP/cc-pVDZ level of theory, using the CTOCDDZ method. It is shown that angular (respectively linear) benzoannelation increases (respectively decreases) the intensity of paratropic (antiaromatic) current density along the four-membered ring of biphenylene. The opposite effect is found for benzocyclobutadieno-annelation. It is shown that the extent of local aromaticity of the four-membered ring in biphenylene congeners can vary from highly antiaromatic to nonaromatic by applying different modes of annelation.
\end{abstract}

\section{Introduction}

Biphenylene and structurally related [n]phenylenes are of significant interest to experimental and theoretical chemistry, because these conjugated systems contain both six-membered rings (likely causing bond-length equalization and aromatic stabilization) and four-membered rings (likely causing doublebond fixation and antiaromatic destabilization). Numerous [n]phenylenes have been synthesized so $\operatorname{far}^{[1-5]}$ and their aromaticity/antiaromaticity has been scrutinized. ${ }^{[6-8]}$ In addition, these compounds are of considerable interest from a photophysical and spectroscopic point of view. ${ }^{[9]}$

Several effects govern the degree of aromaticity/antiaromaticity in conjugated polycyclic compounds, including [n]phenylenes. ${ }^{[10-14]}$ The most intriguing is the effect of different benzo-annelation (B-annelation) modes. ${ }^{[15-17]}$ Already in 2004, it was found that the extent of cyclic conjugation of the central six-membered ring in polybenzoperylenes is significantly influenced by the presence of annelated benzene rings. ${ }^{[18]}$ Later these effects were found to occur rather generally in benzenoid ${ }^{[15,16]}$ and non-benzenoid polycyclic conjugated hydrocarbons. ${ }^{[19]}$ Attaching a benzene ring to a polycyclic conjugated molecule

[a] Dr. S. Radenković, J. Tošović

Faculty of Science, University of Kragujevac, Radoja Domanovića 12, 34000 Kragujevac, Serbia

E-mail: slavkoradenkovic@kg.ac.rs

[b] Prof. R. W. A. Havenith

Theoretical Chemistry, Zernike Institute for Advanced Materials and Stratingh Institute for Chemistry, Rijksuniversiteit Groningen,

Nijenborgh 4, 9747 AG Groningen, The Netherlands

[c] Prof. R. W. A. Havenith, Prof. P. Bultinck

Department of Inorganic and Physical Chemistry, Ghent University, Krijgslaan 281, 9000 Gent, Belgium

Supporting information for this article is given via a link at the end of the document. can be performed in two different ways relative to a given ring $\boldsymbol{R}$ : either in linear or in angular position. If two benzene rings are both angularly annelated to the same benzene ring, then these are said to be geminal with regard to ring $\boldsymbol{R}$. These modes of Bannelations are depicted in Figure 1. In the present paper annelated biphenylene derivates are considered and $\boldsymbol{R}$ is the central four-membered ring in biphenylene (Figure 2). For this reason, only the results referring to the case where the size of $\boldsymbol{R}$ is divisible by four are discussed, although there are more results obtained in the previous studies. ${ }^{[15-19]}$ The following regularities have been found at the Hückel level of theory: ${ }^{[16]}$

Regularity 1B: If ring $\boldsymbol{R}$ has size (i.e., number of atoms) $4 n$ with $n$ an integer, angular and geminal $B$-annelations increase the local antiaromatic character of ring $\boldsymbol{R}$ compared to the same ring in the parent compound.

Regularity $2 B$ : If ring $\boldsymbol{R}$ has size (i.e., number of atoms) $4 n$ with $n$ an integer, linear $B$-annelation decreases the local antiaromatic character of ring $\boldsymbol{R}$ compared to the same ring in the parent compound.

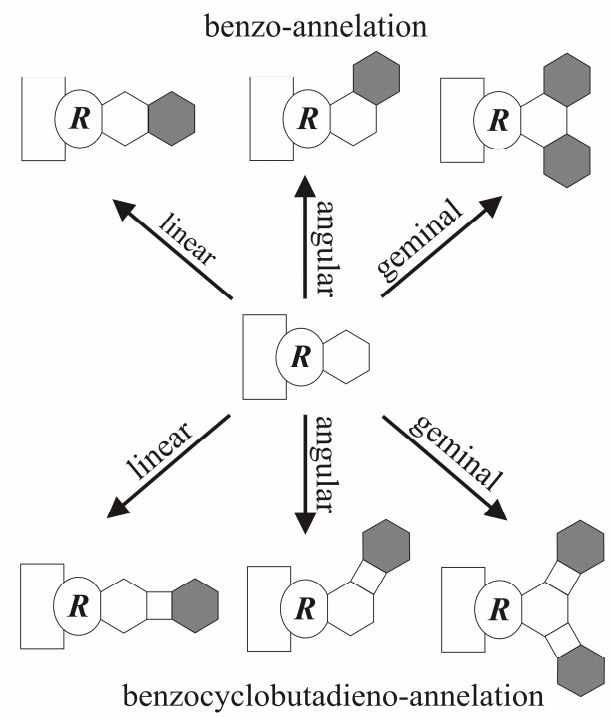

Figure 1. Two types of annelation of a benzene ring to a polycyclic molecules (benzo- and benzocyclobutadieno-annelation) and the three modes of the annelation relative to ring $\boldsymbol{R}$ : linear, angular and geminal.

A different way of attaching a benzene-ring to a polycyclic molecule, referred to as benzocyclobutadieno-annelation (BCBD-annelation), has been investigated. Such annelation is 
also illustrated in Figure 1. In previous works, cyclobutadienoand benzocyclobutadieno-annelation have been shown to be a useful strategy to induce alternation of the $\mathrm{C}-\mathrm{C}$ bond lengths in benzene. ${ }^{[20-25]}$ Compared to the previous case, opposite regularities hold for BCBD-annelation: ${ }^{[17]}$

Regularity $1 B C B D$ : If ring $\boldsymbol{R}$ has size (i.e., number of atoms) $4 n$ with $n$ an integer, angular and geminal BCBDannelations decrease the local antiaromatic character of ring $\boldsymbol{R}$ compared to the same ring in the parent compound.

Regularity 2BCBD: If ring $\boldsymbol{R}$ has size (i.e., number of atoms) $4 n$ with $n$ an integer, linear BCBD-annelation increases the local antiaromatic character of ring $\boldsymbol{R}$ compared to the same ring in the parent compound.

The regularities described above were derived from energy-effect (ef) values calculated using simple Hückel molecular orbital theory. ${ }^{[18]}$ The ef-value of a circuit quantifies the energy-effect caused by cyclic conjugation of m-electrons along the given circuit. Details on this method are outlined in ref ${ }^{[26]}$. Eventually, these regularities were confirmed in a number of other theoretical studies using geometrical and electron delocalization indices of aromaticity. ${ }^{[15,17,27-29]}$ Note that the obtained regularities have never been tested for magnetic indices of aromaticity. This is the main subject of this paper: do magnetic indices confirm these trends or do they rather contradict them?

In the present study current density maps were calculated at the B3LYP level of theory with the diamagnetic-zero (DZ) variant of the continuous transformation of origin of current density (CTOCD) method. ${ }^{[30-33]}$ In the CTOCD-DZ method, also known as the ipsocentric method, ${ }^{[34,35]}$ a distributed origin of the vector potential is used and the current density at each point in space is calculated by choosing itself as the origin of the vector potential. To our knowledge, current density maps of [n]phenylenes have only been recently examined for the first time. ${ }^{[36]}$ In addition, antiaromaticity is much less studied and much less understood compared to aromaticity. In view of this, examining the suspected aromatic character of the sixmembered rings and suspected antiaromatic character of the four-membered rings in phenylenes is an intriguing topic. In this paper a detailed study of the current density maps obtained for B- and BCBD-annelated biphenylene derivates (Figure 2) is presented. This way, we aim to assess the effect of $B$ - and BCBD-annelation on the current density distribution in a series of annelated biphenylene molecules.

\section{Computational details}

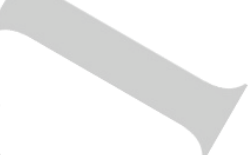

The structures of the examined molecules were optimized at the B3LYP/cc-pVDZ level of theory using the Gaussian 09 program. ${ }^{[37]}$ The optimized geometries of biphenylene and all its considered derivatives were found to be perfectly planar. Hessian calculations showed that the planar geometries of the examined molecules correspond to minima on the potential energy surface at this level of theory. The main reason to use the B3LYP method is that for strongly conjugated hydrocarbon molecules the computed structures are known to be close to

$\operatorname{CCSD}(T)$ results. ${ }^{[38]}$ Note also that the DFT/B3LYP approach has also been used for optimizing geometries in the benchmark W1 thermochemical protocol for very accurate thermochemical calculations. ${ }^{[39]}$

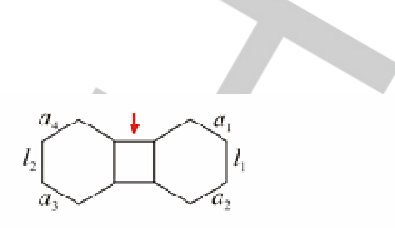

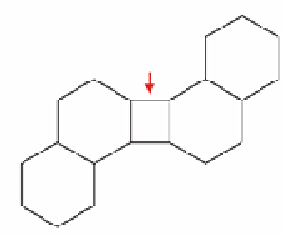

B- $a_{1} a_{3}$

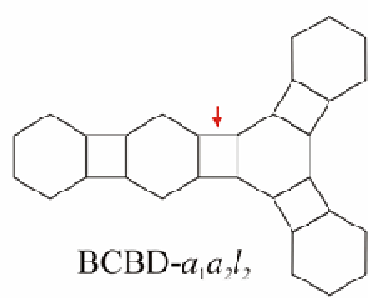

Figure 2. Biphenylene and the labeling of its annelation sites ( $a=$ angular, $I=$ linear with regard to the central four-membered ring). The B- and BCBDannelated biphenylenes are indicated by the sites of annelation. $J_{\max }$ values were calculated for the outer carbon-carbon bonds (indicated by arrows) that belong to the central four-membered ring in the examined molecules.

The current density calculations were performed at the B3LYP/cc-pVDZ level of theory using the CTOCD-DZ method. ${ }^{[30-35,40]}$ In all calculations a unit magnetic field perpendicular to the molecular plane was used and the calculated ring currents were plotted on a grid in the plane parallel to the molecular plane with a diatropic or aromatic current represented by a counterclockwise circulation and a paratropic or antiaromatic one represented by a clockwise circulation. The plotting planes were chosen at $1 \mathrm{a}_{0}$ above the molecular plane.

In order to obtain more quantitative information about the current density distribution in a molecule of interest, the largest magnitude of the current density through a cross section of a bond $\left(J_{\max }\right)$ was employed in this work. $J_{\max }$ can be used as a measure of the bond current strength and was successfully applied in a series of recent studies. ${ }^{[41-43]}$

Calculations of ring currents were performed using inhouse Fortran routines, GAMESS-UK ${ }^{[44]}$ and SYSMO ${ }^{[45]}$.

\section{Results and Discussion}

Total and $\pi$-electron current density maps of biphenylene calculated at the CTOCD-DZ/B3LYP/cc-pVDZ//B3LYP/cc-pVDZ level of theory are presented in Figure 3. The current density maps of biphenylene (Figure 3) clearly indicate the existence of a strong paratropic (antiaromatic) circulation within the fourmembered ring and a somewhat weaker diatropic (aromatic) circulation along the six-membered rings. The current density distribution in biphenylene is in agreement with a naive application of a local version of Hückel $4 n+2$ rule if we assume that the four-membered ring harbours $4 \pi$ electrons and the 
benzene rings 6 . It is a noteworthy fact that biphenylene is a stable molecule, although it sustains such a strong paratropic current density which is usually related to thermodynamic destabilization. A significant advantage of the CTOCD-DZ method is that the total current density can be partitioned into molecular orbital contributions. ${ }^{[34,35]}$ The contribution of an occupied orbital is determined by the accessibility of the unoccupied orbitals by rotational and translational transitions. The contribution of the given transition becomes more important as the energy difference between the occupied and virtual orbital is smaller. Rotational transitions lead to paratropic (antiaromatic) circulation, whereas translational transitions lead to diamagnetic (aromatic) circulation. Figure 3 presents orbital current densities of the highest four occupied molecular orbitals in biphenylene. It can be seen that the $\pi-\mathrm{HOMO}\left(2 b_{2 g}\right)$ current density has the most significant contribution to the total current density, and practically completely determines the current density distribution in the central four-membered ring. The orbital current densities of HOMO-1 $\left(1 a_{\mathrm{u}}\right)$ and HOMO-3 (2 $\left.2 b_{3 \mathrm{u}}\right)$ contribute to the diatropic current density along the molecular perimeter. The orbital current density of the HOMO-2 $\left(1 b_{1 \mathrm{~g}}\right)$ level has only minor contributions to the paratropic circulation along the four- membered ring, as well as to the diatropic circulation along the six-membered rings.

In order to examine the effect of the two different types of annelation (Figure 1) all possible B- and BCBD-annelated biphenylene derivates were considered. In order to simplify notation, the sites of annelation in biphenylene are labeled as indicated in Figure 2. The total current density maps of two Bannelated (B- $l_{1} l_{2}$ and $\left.B-a_{1} a_{2} a_{3} a_{4}\right)$ and two BCBD-annelated biphenylene derivates (BCBD- $l_{1} l_{2}$ and BCBD- $\left.a_{1} a_{2} a_{3} a_{4}\right)$ are presented in Figure 4. The total and $\pi$-electron current density maps of all other examined molecules are given in the Supporting information. The main goal of this work is to examine the changes of the induced current density along the central four-membered ring of biphenylene upon annelation. From Figure 4, as well as from Figures S1 and S2 (see Supporting information), it is evident that the current density distribution along the central four-membered ring of biphenylene derivates depends significantly on the number and position of B- and BCBD-annelations.

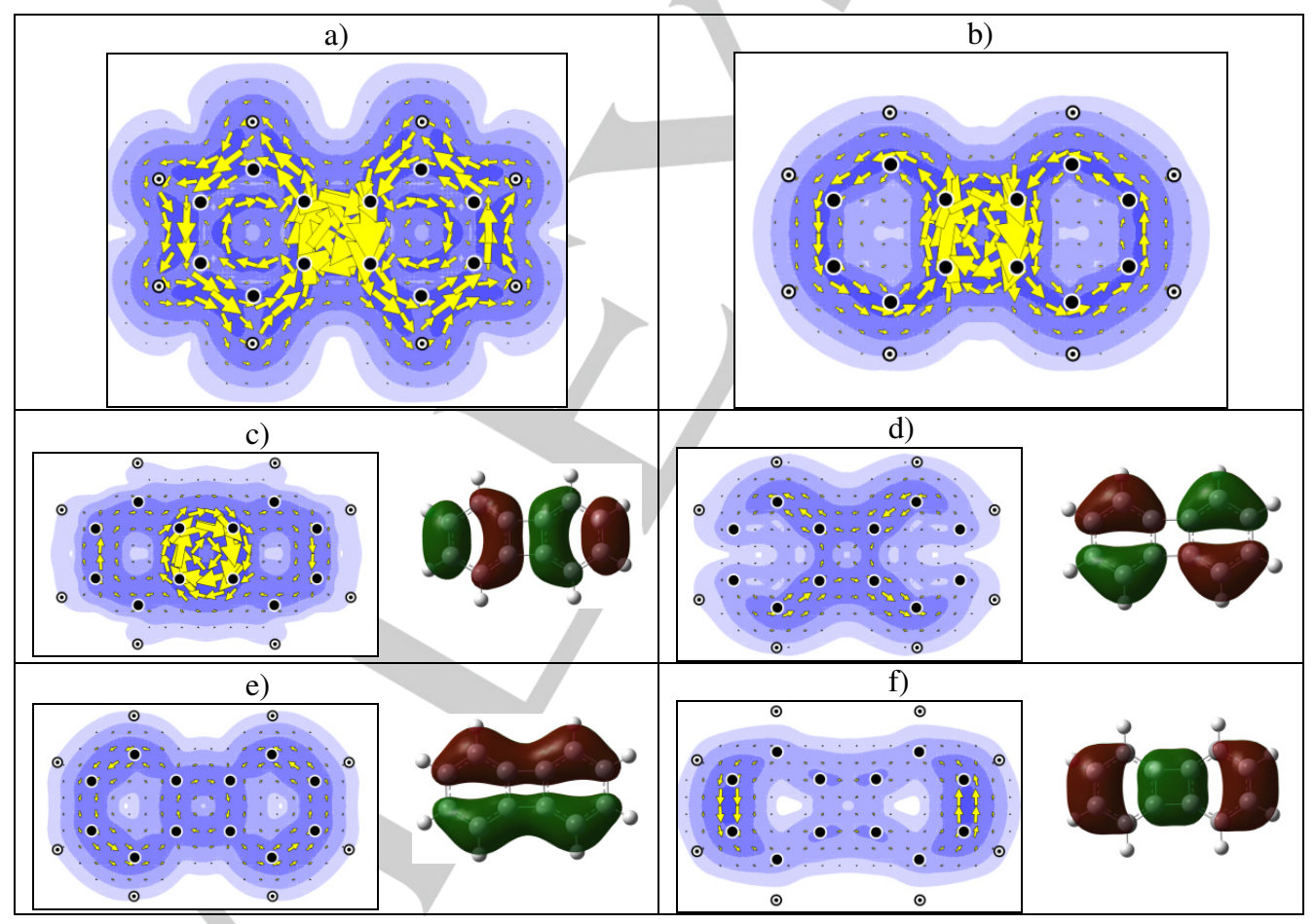

Figure 3. Current density maps of biphenylene obtained with the CTOCD-DZ/B3LYP/cc-pVDZ method: total $(\sigma+\pi)$ current density (a), $\pi$-electron current density (b), HOMO $\left(2 b_{2 g}\right)$ current density (c), HOMO-1 $\left(1 a_{u}\right)$ current density (d), HOMO-2 $\left(1 b_{1 g}\right)$ current density (e) and HOMO-3 $\left(2 b_{3 u}\right)$ current density (f). $\mathrm{H}$ and C atoms are represented by dotted and filled circles, respectively. 


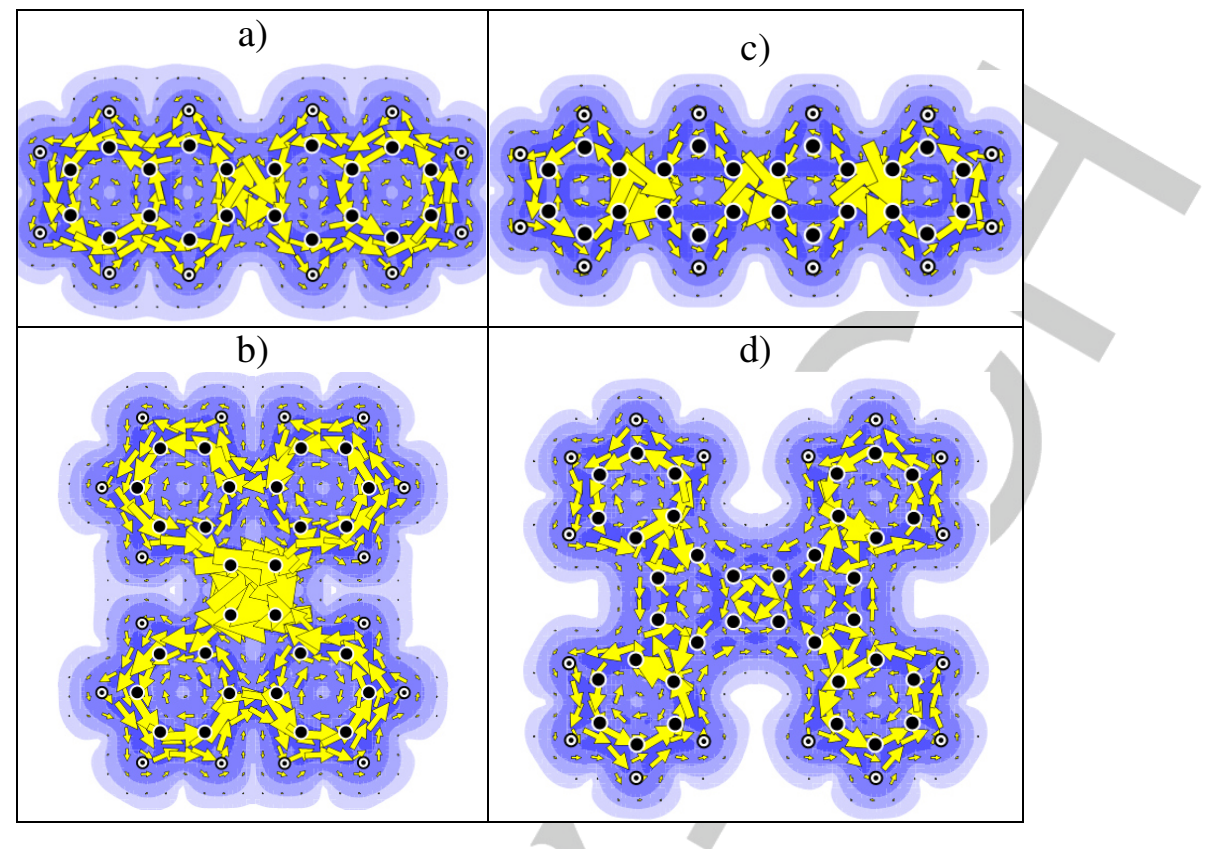

Figure 4. Total current density maps obtained with the CTOCD-DZ/B3LYP/cc-pVDZ method for two benzo-annelated biphenylenes: B- $l_{1} l_{2}(a), B-a_{1} a_{2} a_{3} a_{4}(b)$ and two benzocyclobutadieno-annelated biphenylenes: BCBD- $l_{1} l_{2}(c), B C B D-a_{1} a_{2} a_{3} a_{4}(d)$.

Table 1. Largest magnitudes $\left(J_{\max }\right)$ of the $\pi$-electron current density (expressed relative to the $J_{\text {max }}$-value of cyclobutadiene) through the cross sections of the outer carbon-carbon bond of the central four-membered ring in B- and BCBD-annelated biphenylenes (Figure 2).

\begin{tabular}{ccccccc}
\hline compound & $J_{\max }$ & compound & $J_{\max }$ & $\mathrm{A}$ & $\mathrm{L}$ & $\mathrm{G}$ \\
\hline biphenylene & 0.630 & & & 0 & 0 & 0 \\
B- $l_{1}$ & 0.491 & BCBD- $l_{1}$ & 0.639 & 0 & 1 & 0 \\
B- $a_{1}$ & 0.796 & BCBD- $a_{1}$ & 0.380 & 1 & 0 & 0 \\
B- $l_{1} l_{2}$ & 0.380 & BCBD- $l_{1} l_{2}$ & 0.593 & 0 & 2 & 0 \\
B- $a_{1} l_{2}$ & 0.611 & BCBD- $a_{1} l_{2}$ & 0.343 & 1 & 1 & 0 \\
B- $a_{1} a_{2}$ & 0.833 & BCBD- $a_{1} a_{2}$ & 0.194 & 0 & 0 & 2 \\
B- $a_{1} a_{3}$ & 1.046 & BCBD $-a_{1} a_{3}$ & 0.250 & 2 & 0 & 0 \\
B- $a_{1} a_{4}$ & 1.065 & BCBD- $a_{1} a_{4}$ & 0.250 & 2 & 0 & 0 \\
B- $a_{1} a_{2} l_{2}$ & 0.639 & BCBD $-a_{1} a_{2} l_{2}$ & 0.139 & 0 & 1 & 2 \\
B- $a_{1} a_{2} a_{3}$ & 1.130 & BCBD- $a_{1} a_{2} a_{3}$ & 0.139 & 1 & 0 & 2 \\
B- $a_{1} a_{2} a_{3} a_{4}$ & 1.222 & BCBD- $a_{1} a_{2} a_{3} a_{4}$ & 0.111 & 0 & 0 & 4 \\
\hline
\end{tabular}

In order to obtain a quantitative measure of the current density along the central four-membered ring, the $J_{\max }$ of the outer carbon-carbon bond that belongs to this ring was examined (Figure 2). $J_{\max }$ thus serves as a measure of the intensity of the induced current density along the central fourmembered ring in the given molecule. $J_{\max }$ values of the outer carbon-carbon bond that belongs to the central four-membered ring of the studied systems are given in Table 1. $J_{\max }$ values for all carbon-carbon bonds in the examined molecules are presented in Figures S3 and S4 (Supporting information). The number of annelated rings attached by angular-, linear- and geminal-annelation are indicated by $A, L$ and $G$, respectively (Table 1). In the case of angular and linear annelation one new ring is attached to the peripheral hexagonal ring, and in the case of geminal annelation, two new rings are attached to the peripheral hexagonal ring of biphenylene (Figure 1).

In what follows the data from Table 1 is used to scrutinize the validity of the different regularities. For the $\mathrm{B}$-annelation, the data show that both regularities $1 \mathrm{~B}$ and $2 \mathrm{~B}$ are satisfied. This can be illustrated by the following data: $J_{\max }=0.630$ for biphenylene (no annelation); $J_{\max }=0.796$ for $\mathrm{B}-\mathrm{a}_{1}$ (one angular B-annelation); $J_{\max }=0.491$ for $\mathrm{B}-l_{1}$ (one linear B-annelation). These examples indicate that angular (respectively linear) Bannelation increases (respectively decreases) the magnitude of paratropic (antiaromatic) circulation within the central fourmembered ring. The effects of angular and linear annelations have about the same intensity, as seen from $J_{\max }=0.611$ for B$a_{1} l_{1}$ (one angular and one linear B-annelation). In addition, the effect of B-annelation is proportional to the number of the attached benzene rings. For instance, $J_{\max }=0.833$ for $\mathrm{B}-\mathrm{a}_{1} a_{2}$ (two angular B-annelations); $J_{\max }=1.130$ for $\mathrm{B}-\mathrm{a}_{1} a_{2} a_{3}$ (three angular B-annelations); $J_{\max }=1.222$ for $\mathrm{B}-\mathrm{a}_{1} a_{2} a_{3} a_{4}$ (four angular B-annelations), and $J_{\max }=0.380$ for $B-l_{1} l_{2}$ (two linear Bannelations). 

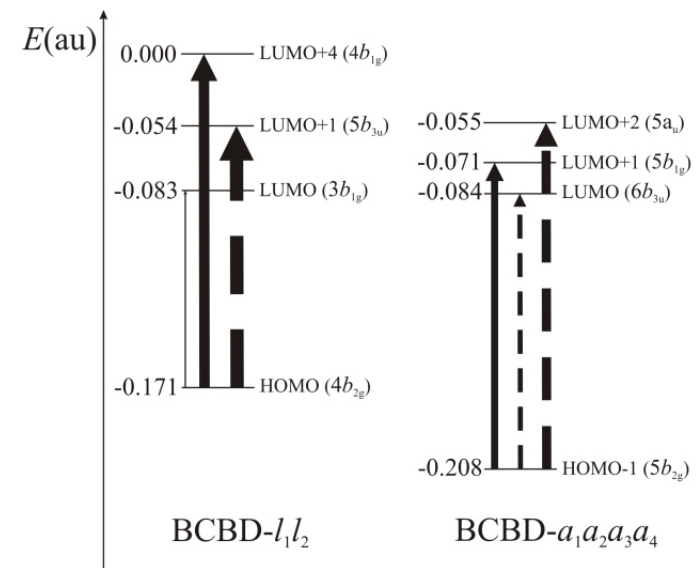

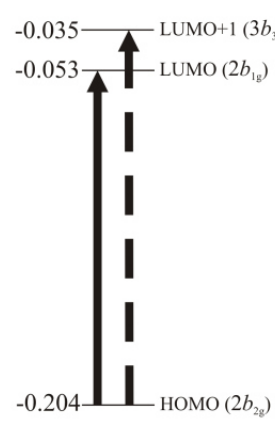

biphenylene

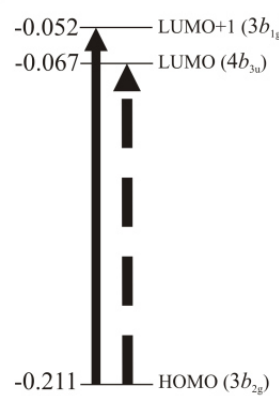

$\mathrm{B}-l_{1} l_{2}$

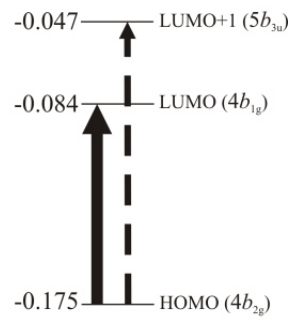

B- $a_{1} a_{2} a_{3} a_{4}$

Figure 5. Frontier orbital energy levels (in au) of biphenylene, BCBD- $l_{1} l_{2}$, BCBD- $a_{1} a_{2} a_{3} a_{4}, B-l_{1} l_{2}$ and B- $a_{1} a_{2} a_{3} a_{4}$ obtained at the B3LYP/cc-pVDZ level of theory. Full arrows represent the main rotational (paratropic) transitions, and dashed arrows represent the main translational (diatropic) transitions. The width of arrows reflects the magnitude of the contribution of the given transition relative to the analogous one in biphenylene (for details see ref. ${ }^{[34]}$ ).

A different picture is found for BCBD annelation. Regularity $1 B C B D$ is found to be valid throughout, but regularity $2 B C B D$ is not. Regularity $1 \mathrm{BCBD}$ is illustrated by the following result: $J_{\max }=$ 0.630 for biphenylene (no annelation) and $J_{\max }=0.380$ for BCBD- $a_{1}$ (one angular BCBD-annelation). This implies that the effect of BCBD-annelation is opposite to the analogous effect of $\mathrm{B}$-annelation: angular BCBD-annelation decreases the intensity of paratropic (antiaromatic) circulation within the four-membered ring. The effect of angular BCBD-annelation is proportional to the number of the annelated rings. This is convincingly clear from the following data: $J_{\max }=0.194$ for BCBD- $a_{1} a_{2}$ (two angular BCBD-annelations); $J_{\max }=0.139$ for BCBD- $a_{1} a_{2} a_{3}$ (three angular BCBD-annelations); $J_{\max }=0.111$ for BCBD- $a_{1} a_{2} a_{3} a_{4}$ (four angular BCBD-annelations). The obtained results (Table 1) are not in agreement with regularity $2 \mathrm{BCBD}$, although it can be seen that the effect of linear BCBD-annelation on the current density induced in the four-membered ring of biphenylene is rather negligible. As seen from Table 1, the paratropic current densities induced along the four-membered ring in biphenylene $\left(J_{\max }=\right.$ $0.630)$, BCBD- $I_{1}\left(J_{\max }=0.639\right)$ and BCBD- $I_{1} I_{2}\left(J_{\max }=0.593\right)$ have practically the same intensity. With the exception of BCBD- $l_{1}$, all BCBD-derivatives have weaker intensity of paratropic currents within the central four-membered ring than biphenylene.

Regularities stated above give a qualitative description of the effect of B- and BCBD-annelations. The data from Table 1 show that the $J_{\max }$-values differ slightly for isomers with the same values for $A, L$, and $G$. In ref ${ }^{[15]}$ it has been shown that between the ef-values and the position and number of $\mathrm{B}$ annelated rings exists a quantitative relation. By applying the same methodology, it is now found that the intensity of the current density induced within the four-membered ring, measured through the $J_{\text {max }}-$ values can be expressed as a linear function of $A, L$ and $G$ numbers, indicating the numbers of $B$ and BCBD-annelations in angular, linear and geminal position, respectively. The following relations for $\mathrm{B}$ - and $\mathrm{BCBD}$-annelated biphenylene derivates were obtained, respectively:

$$
\begin{gathered}
J_{\max }(B)=(0.22 \pm 0.03) A-(0.15 \pm 0.04) L \\
+(0.14 \pm 0.02) G+(0.61 \pm 0.05)
\end{gathered}
$$

$$
\begin{gathered}
J_{\max }(B C B D)=-(0.18 \pm 0.04) A-(0.03 \pm 0.05) L \\
-(0.15 \pm 0.03) G+(0.60 \pm 0.07)
\end{gathered}
$$

The parameters that appear in Eqs. 1 and 2 were determined by least-squares fitting for the sets of $11 \mathrm{~B}$-annelated and 11 BCBD-annelated biphenylene derivates, respectively. The respective correlation coefficients are 0.9817 and 0.9324 .

It is found that in the case of B-annelation, the coefficient of $G$ in Eq. (1) is lower than the coefficient of $A$. This implies that two angular B-annelations on the same ring have a significantly weaker influence on the intensity of cyclic conjugation in the central four-membered ring compared to the effect of two angular annelations on different rings.

In order to assess the basis set effect, the current density maps were calculated using the minimal STO-3G basis set. The plot of the $J_{\max }$ values of the outer carbon-carbon bond of the central four-membered ring in B- and BCBD-annelated biphenylenes calculated with the cc-pVDZ basis set versus the respective values obtained with the STO-3G basis set is shown in Figure S5 (see Supporting information). As seen from Figure S5 there is a good linear correlation between the two $J_{\max }$-values. This is in agreement with the previous works showing that a minimal basis set provides essentially the same information about the calculated current densities as other larger basis sets. ${ }^{[46,47]}$ In addition, it has been shown that even the pseudo- $\pi$ method can provide the necessary information about the current density distribution ${ }^{[48]}$ in polycyclic conjugated hydrocarbons. 
Figure S6 (see Supporting information) shows that there exist a reasonably good, but evidently non-linear correlation between the $J_{\max }$-values of $\pi$-electron and total $(\pi+\sigma)$ current density. This implies that B- and BCBD-annelations affect in a similar way both $\sigma$ - and $\pi$-electronic systems of the studied molecules.

As seen from Figure 3 , in biphenylene the main contribution to the paratropic current density induced in the central four-membered ring comes from the HOMO $\left(2 b_{2 g}\right)$ level. There are two relevant transitions from the HOMO $\left(2 b_{2 g}\right)$ level in biphenylene: rotational transition to the LUMO $\left(2 b_{1 \mathrm{~g}}\right)$ level and translational transition to the LUMO+1 $\left(3 b_{3 \mathrm{u}}\right)$ level (Figure 5). The latter weakens the overall paratropic contribution of the HOMO to the total current density. By inspection of the orbital current densities, it was found that the main part of paratropic circulations within the central four-membered ring of $B$ - and BCBD-annelated biphenylenes comes from a single frontier orbital current density. Figure 5 presents analogous transitions relevant for the current density induced in the central fourmembered ring of B- $l_{1} l_{2}, B-a_{1} a_{2} a_{3} a_{4}, B C B D-l_{1} l_{2}$ and BCBD$a_{1} a_{2} a_{3} a_{4}$. Note that the paratropic current within the central fourmembered ring of B- $l_{1} l_{2}, B C B D-l_{1} l_{2}, B C B D-a_{1} a_{2} a_{3} a_{4}$ is weaker, and of $B-a_{1} a_{2} a_{3} a_{4}$ is stronger compared to the analogous current in biphenylene (Table 1). The data from Figure 5 show that in $B-$ $l_{1} l_{2}$ the LUMO $\left(4 b_{3 u}\right)$ level is energetically more accessible for the translational transition from the HOMO $\left(3 b_{2 g}\right)$ level than the LUMO+1 $\left(3 b_{1 g}\right)$ level for the corresponding rotational transition. The magnitude of these transitions relative to the magnitude of the analogous transitions in biphenylene reveals that the overall paratropic HOMO current density is weaker in $\mathrm{B}-\mathrm{l}_{1} l_{2}$ than in biphenylene. The energy differences between the HOMO $\left(4 b_{2 g}\right)$ and LUMO $\left(4 b_{1 \mathrm{~g}}\right)$ and between the HOMO $\left(4 b_{2 \mathrm{~g}}\right)$ and LUMO+1 $\left(5 b_{3 u}\right)$ in $\mathrm{B}-a_{1} a_{2} a_{3} a_{4}$ are smaller compared to the analogous energy differences in biphenylene. The relative magnitude of the contribution of the respective transitions shows that the paratropic $\mathrm{HOMO}$ current density in $\mathrm{B}-\mathrm{a}_{1} a_{2} a_{3} a_{4}$ is stronger than in biphenylene. The relative contributions of the rotational transition HOMO $\left(4 b_{2 g}\right)$ to LUMO $\left(3 b_{1 g}\right)$ in BCBD- $l_{1} l_{2}$ and translational transition HOMO-1 $\left(5 b_{2 g}\right)$ to LUMO $\left(6 b_{3 u}\right)$ in BCBD$a_{1} a_{2} a_{3} a_{4}$ are remarkably smaller than the corresponding ones in biphenylene. On the other hand, there are two additional relevant transitions in these molecules: rotational transition $\mathrm{HOMO}\left(4 b_{2 g}\right)$ to LUMO+4 $\left(4 b_{1 \mathrm{~g}}\right)$ in BCBD- $l_{1} l_{2}$ and translational transition HOMO-1 $\left(5 b_{2 g}\right)$ to LUMO+2 $\left(5 a_{\mathrm{u}}\right)$ in BCBD- $a_{1} a_{2} a_{3} a_{4}$. The relative contribution of the relevant transitions shows that the paratropic current density in the central ring of BCBD- $I_{1} I_{2}$ and BCBD- $a_{1} a_{2} a_{3} a_{4}$ are weaker than the one in biphenylene.

\section{Conclusions}

The effect of B- and BCBD-annelation on the local aromaticity of the central four-membered ring in annelated biphenylenes was examined by means of the induced current density, calculated at the CTCD-DZ/B3LYP/cc-pVDZ// B3LYP/cc-pVDZ level of theory. This way, the effect of different modes of annelation was tested using magnetic indicators of aromaticity. It is shown that angular (respectively linear) B-annelation increases (respectively decreases) the magnitude of paratropic (antiaromatic) current density along the four-membered ring of biphenylene. The analogous effect of BCBD-annelation is found to be opposite to the effect of B-annelation. It is shown that the effect of B- and BCBD-annelation can be well represented by a linear function of the number of angular, linear and geminal annelations. These results are in agreement with the regularities found with other indices of aromaticity. It is found that the local aromaticity of the four-membered ring in biphenylene congeners can vary from highly antiaromatic to nonaromatic by applying different types of annelation. The effect of different annelation modes can be used for tuning the local aromaticity to the desired degree.

\section{Acknowledgements}

This work is supported by the Ministry of Science of Serbia (Grant No 174033). RWAH acknowledges Prof. Dr. R. Broer (University of Groningen, The Netherlands) for fruitful discussions and the Zernike Institute for Advanced Materials of the University of Groningen for financial support (Dieptestrategie program). The work was partially completed with computing time at the Dutch National Supercomputer Huygens (SURFsara, SH213-11).

Keywords: magnetic properties $\cdot$ density functional calculations - aromaticity $\bullet$ polycycles $\bullet$ hydrocarbons

[1] M. Hirthammer, K. P. C. Vollhardt, J. Am. Chem. Soc. 1986, 108, 2481-2482.

[2] C. Eickmeier, D. Holmes, H. Junga, A. J. Matzger, F. Scherhag, M. Shim, K. P. C. Vollhardt, Angew. Chemie Int. Ed. 1999, 38, 800-804.

[3] S. Han, A. D. Bond, R. L. Disch, D. Holmes, J. M. Schulman, S. J. Teat, K. P. C. Vollhardt, G. D. Whitener, Angew. Chemie Int. Ed. 2002, 41, 3223-3227.

[4] D. Bruns, H. Miura, K. P. C. Vollhardt, A. Stanger, Org. Lett. 2003, 5, 549-552.

[5] B. Iglesias, A. Cobas, D. Pérez, E. Guitián, K. P. C. Vollhardt, Org. Lett. 2004, 6, 3557-3560.

[6] Z. B. Maksić, D. Kovacek, M. Eckert-Maksić, M. Böckmann, M. Klessinger, J. Phys. Chem. 1995, 99, 6410-6416.

[7] J. M. Schulman, R. L. Disch, H. Jiao, P. von R. Schleyer, J. Phys. Chem. A 1998, 102, 8051-8055.

[8] M. Randić, A. T. Balaban, D. Plavšić, Phys. Chem. Chem. Phys. 2011, 13, 20644-20648.

[9] C. Dosche, H.-G. Lohmannsroben, A. Bieser, P. I. Dosa, S. Han, M. Iwamoto, A. Schleifenbaum, K. P. C. Vollhardt, Phys. Chem. Chem. Phys. 2002, 4, 2156-2161.

[10] R. W. A. Havenith, P. W. Fowler, S. Fias, P. Bultinck, Tetrahedron Lett. 2008, 49, 1421-1424.

[11] W. P. Oziminski, T. M. Krygowski, P. W. Fowler, A. Soncini, Org. Lett. 2010, 12, 4880-4883.

[12] M. Kaipio, M. Patzschke, H. Fliegl, F. Pichierri, D. Sundholm, J. Phys. Chem. A 2012, 116, 10257-10268.

[13] T. M. Krygowski, K. Ejsmont, B. T. Stepień, M. K. Cyrański, J. Poater, M. Solà, J. Org. Chem. 2004, 69, 6634-6640.

[14] J. O. C. Jiménez-Halla, E. Matito, L. Blancafort, J. Robles, M. Solà, J. Comput. Chem. 2009, 30, 2764-2776.

[15] A. T. Balaban, J. Đurđević, I. Gutman, S. Jeremić, S. Radenković, J. Phys. Chem. A 2010, 114, 5870-5877. 
[16] A. Balaban, I. Gutman, S. Jeremić, J. Đurđević, Monatshefte für Chemie - Chem. Mon. 2011, 142, 53-57.

[17] A. Balaban, I. Gutman, S. Marković, D. Simijonović, Monatshefte für Chemie - Chem. Mon. 2011, 142, 797-800.

[18] I. Gutman, N. Turković, J. Jovičić, Monatshefte für Chemie - Chem. Mon. 2004, 135, 1389-1394.

[19] S. Radenković, J. Đurđević, I. Gutman, Chem. Phys. Lett. 2009, 475, 289-292.

[20] P. W. Fowler, R. W. A. Havenith, L. W. Jenneskens, A. Soncini, E. Steiner, Chem. Commun. 2001, 2386-2387.

[21] A. Soncini, R. W. A. Havenith, P. W. Fowler, L. W. Jenneskens, E. Steiner, J. Org. Chem. 2002, 67, 4753-4758.

[22] G. J. Hill, P. B. Karadakov, D. L. Cooper, Mol. Phys. 2006, 104, 677680.

[23] P. Bultinck, M. Mandado, R. Mosquera, J. Math. Chem. 2008, 43, 111118.

[24] A. Stanger, J. Phys. Chem. A 2008, 112, 12849-12854.

[25] R. W. A. Havenith, L. W. Jenneskens, P. W. Fowler, Chem. Phys. Lett. 2003, 367, 468-474.

[26] I. Gutman, Monatshefte für Chemie - Chem. Mon. 2005, 136, 10551069.

[27] J. Đurđević, S. Radenković, I. Gutman, S. Marković, Monatshefte für Chemie - Chem. Mon. 2009, 140, 1305-1309.

[28] I. Gutman, S. Radenković, W. Linert, Monatshefte für Chemie - Chem. Mon. 2010, 141, 401-407.

[29] J. Đurđević, I. Gutman, R. Ponec, J. Serb. Chem.Soc. 2009, 74, 549554

[30] T. A. Keith, R. F. W. Bader, Chem. Phys. Lett. 1993, 210, 223-231.

[31] T. A. Keith, R. F. W. Bader, J. Chem. Phys. 1993, 99, 3669-3682.

[32] P. Lazzeretti, M. Malagoli, R. Zanasi, Chem. Phys. Lett. 1994, 220, 299-304.

[33] R. Zanasi, J. Chem. Phys. 1996, 105, 1460-1469.

[34] E. Steiner, P. W. Fowler, J. Phys. Chem. A 2001, 105, 9553-9562.
[35] E. Steiner, P. W. Fowler, Chem. Commun. 2001, 2220-2221.

[36] R. Gershoni-Poranne, C. M. Gibson, P. W. Fowler, A. Stanger, J. Org. Chem. 2013, 78, 7544-7553.

[37] M. J. Frisch, G. W. Trucks, H. B. Schlegel, G. E. Scuseria, M. A. Robb, J. R. Cheeseman, G. Scalmani, V. Barone, B. Mennucci, G. A Petersson, et al., Gaussian 09, Revis. B.01, Gaussian, Inc., Wallingford CT 2009.

[38] M. S. Deleuze, M. G. Giuffreda, J.-P. François, J. Phys. Chem. A 2002, 106, 5626-5637

[39] J. M. L. Martin, G. de Oliveira, J. Chem. Phys. 1999, 111, 1843-1856.

[40] R. W. A. Havenith, P. W. Fowler, Chem. Phys. Lett. 2007, 449, 347353.

[41] J. Jusélius, D. Sundholm, J. Gauss, J. Chem. Phys. 2004, 121, 3952 3963.

[42] H. Fliegl, D. Sundholm, S. Taubert, J. Jusélius, W. Klopper, J. Phys. Chem. A 2009, 113, 8668-8676.

[43] G. Monaco, R. Zanasi, S. Pelloni, P. Lazzeretti, J. Chem. Theory Comput. 2010, 6, 3343-3351.

[44] M. F. Guest, I. J. Bush, H. J. J. van Dam, P. Sherwood, J. M. H. Thomas, J. H. van Lenthe, R. W. A. Havenith, J. Kendrick, Mol. Phys. 2005, 103, 719-747.

[45] P. Lazzeretti, R. Zanasi, SYSMO Package, University of Modena, 1980, with additional routines for evaluation and plotting of current density by E. Steiner and P. W. Fowler, R. W. A. Havenith and A. Soncini.

[46] E. Steiner, P. W. Fowler, Int. J. Quantum Chem. 1996, 60, 609-616.

[47] A. Ligabue, U. Pincelli, P. Lazzeretti, R. Zanasi, J. Am. Chem. Soc. 1999, 121, 5513-5518.

[48] P. W. Fowler, E. Steiner, Chem. Phys. Lett. 2002, 364, 259-266. 


\section{Entry for the Table of Contents}

\section{ARTICLE}

The effect of benzo- and benzocyclobutadienoannelation on the current density induced in a series of biphenylene derivates is examined. It is shown that the extent of local aromaticity of the fourmembered ring in biphenylene congeners can vary from highly antiaromatic to nonaromatic by applying different modes of annelation.
Slavko Radenković*, Jelena Tošović, Remco W.A. Havenith, and Patrick Bultinck
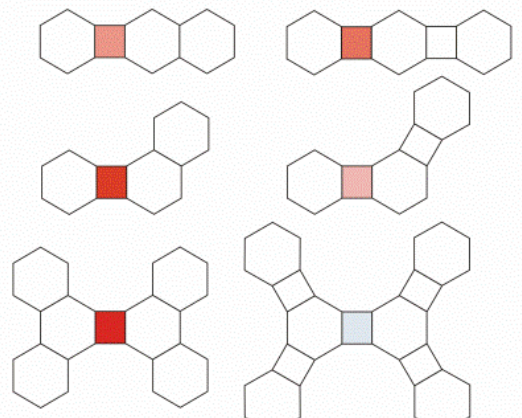

Page No. - Page No.

Ring currents in benzo- and benzocyclobutadieno-annelated biphenylene derivates

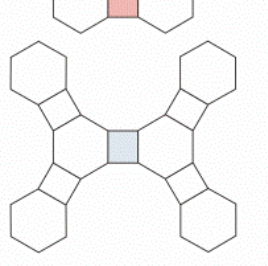

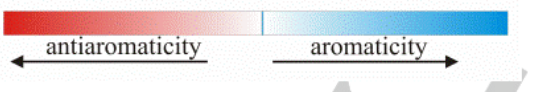

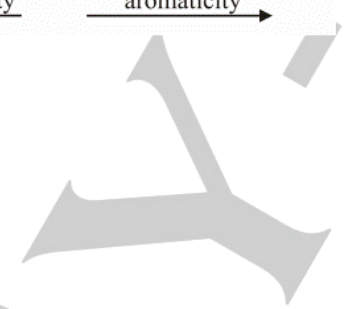

